\title{
MyD88 TIR domain higher-order assembly interactions revealed by serial femtosecond crystallography
}

\author{
Susannah Holmes ${ }^{1,10}$, Max T. B. Clabbers ${ }^{2,10,11}$, Timothy W. Muusse ${ }^{3}$, Parimala R. Vajjhala ${ }^{3}$, Sara J. Thygesen ${ }^{3}$, \\ Alpeshkumar K. Malde ${ }^{4}$, Dominic J. B. Hunter ${ }^{3,5,6}$, Tristan I. Croll ${ }^{7}$, Leonie Flueckiger ${ }^{1}$, Jeffrey D. Nanson ${ }^{3}$, Md. \\ Habibur Rahaman ${ }^{3}$, Andrew Aquila ${ }^{8}$, Mark S. Hunter ${ }^{8}$, Mengning Liang ${ }^{8}$, Chun Hong Yoon ${ }^{8}$, Jingjing Zhao ${ }^{2}$, Nadia \\ A. Zatsepin ${ }^{1}$, Brian Abbey ${ }^{1}$, Emma Sierecki ${ }^{5}$, Yann Gambin ${ }^{5}$, Katryn J. Stacey ${ }^{3,6,9}$, Connie Darmanin ${ }^{1}$, Bostjan \\ $\mathrm{Kobe}^{3,6,9}$, Hongyi $\mathrm{Xu}^{2}$, Thomas $\mathrm{Ve}^{4}$ \\ ${ }^{1}$ Australian Research Council Centre of Excellence in Advanced Molecular Imaging, Department of Chemistry and Physics, La Trobe \\ Institute for Molecular Science, La Trobe University, Melbourne, Victoria, Australia; \\ ${ }^{2}$ Department of Materials and Environmental Chemistry, Stockholm University, Stockholm, Sweden; \\ ${ }^{3}$ School of Chemistry and Molecular Biosciences, The University of Queensland, Brisbane, Queensland, Australia; \\ ${ }^{4}$ Institute for Glycomics, Griffith University, Southport, Queensland, Australia; \\ ${ }^{5}$ EMBL Australia Node in Single Molecule Science, University of New South Wales, Kensington, New South Wales, Australia; \\ ${ }^{6}$ Institute for Molecular Bioscience, The University of Queensland, Brisbane, Queensland, Australia; \\ ${ }^{7}$ Cambridge Institute for Medical Research, University of Cambridge, Cambridge, UK, \\ ${ }^{8}$ Linac Coherent Light Source, SLAC National Accelerator Laboratory, Menlo Park, California, USA; \\ ${ }^{9}$ Australian Infectious Diseases Research Centre, The University of Queensland, Brisbane, Queensland, Australia; \\ ${ }^{10}$ These authors contributed equally: Susannah Holmes, Max T. B. Clabbers; \\ ${ }^{11}$ Present address: Department of Biological Chemistry, University of California Los Angeles, Los Angeles, California, USA;
}

susannahrholmes@gmail.com

MyD88 and MAL are Toll-like receptor (TLR) adaptors that signal to induce pro-inflammatory cytokine production. We previously observed that the TIR domain of MAL (MAL ${ }^{\mathrm{TIR}}$ ) forms filaments in vitro and induces formation of crystalline higher-order assemblies of the MyD88 TIR domain (MyD88 ${ }^{\mathrm{TIR}}$ ). Due to their crystal size, conventional crystallography proved to be challenging. However, through serial femtosecond crystallography (SFX) we were able to determine the structure of MyD88 crystals. Here, we present the SFX structure of the MyD88 $8^{\mathrm{TIR}}$ assembly, which revealed a biological relevant two-stranded higher order assembly arrangement of TIR domains analogous to that seen previously for MAL ${ }^{\text {TIR }}$. Our study provides structural and mechanistic insights into TLR signal transduction ${ }^{1}$.

${ }^{1}$ Clabbers, M., Holmes, S. et.al. MyD88 TIR domain higher-order assembly interactions revealed by microcrystal electron diffraction and serial femtosecond crystallography. Nature Communications, accepted March 2021, DOI: 10.1038/s41467-021-22590-6

Keywords: SFX, MyD88, pro-inflammatory, higher-order 